\begin{tabular}{|l|c|c|c|c|} 
Revue suisse Zool. & Tome 81 & Fasc. 4 & p. 731-735 & Genève, décembre 1974 \\
\hline
\end{tabular}

\title{
Un nuovo Schizomida delle Batu Caves in Malesia (Arachnida, Schizomida)
}

di

\section{Paolo Marcello BRIGNOLI}

Con 9 figure

Dopo le ricerche di Abraham, Fage, Bristowe e Roewer sugli Aracnidi delle famose Batu Caves sembrava quasi impossibile che qualcosa fosse sfuggito, non poca sorpresa ha quindi suscitato in me il rinvenimento di uno Schizomida in una piccola collezione di Aracnidi recentemente raccolti in queste grotte da M. R. Pilet.

Nessuno Schizomida era stato infatti finora ritrovato nelle Batu Caves, anche se la loro presenza non era improbabile, visto che questo gruppo sembra abbastanza frequente nelle grotte tropicali.

Come in generale per gli Schizomidi (assai di rado provvisti di occhi e per lo più depigmentati, anche se non cavernicoli) è difficile dire se questa specie nuova, come era da attendersi, sia cavernicola o no; le sue dimensioni, non indifferenti, non sono eccezionali (simili sono quelle di Trithyreus suboculatus Pocock, 1900 e di T. grassii (Thorell, 1889), limitandosi alle forme asiatiche), notevole è la lunghezza delle zampe I (a funzione tattile), sia assoluta che relativa (di poco inferiore a 2 volte quella del corpo), ma anche questo carattere è di incerto valore.

Ringrazio il Dr. Bernd Hauser (Muséum d'Histoire naturelle de Genève) che con estrema gentilezza mi ha confidato questo individuo facente parte delle collezioni di cui è conservatore.

Trithyreus pileti $\mathrm{n}$. $\mathrm{sp}$.

Materiale esaminato: Malesia, Kuala Lumpur, Batu Caves, Selangor, 24. VII. 69, R. Pilet leg., $1 \hat{o}$ (holotypus). 
Descrizione - $\hat{o}$ (q ignota): prosoma di colore olivastro chiaro; occhi assenti; propeltidio (v. fig. 6) con caratteristica piccola punta anteriore; mesopeltidio ben visibile; metapeltidio nettamente diviso in due metà. I sterno a triangolo, con punta lunga e sottile, separante nettamente le II coxe; II sterno piccolo e stretto, separante di poco, ma nettamente le IV coxe. Cheliceri, v. fig. 2; palpi, v. fig. 1 ; zampe senza peculiarità (estremità del tarso I v. fig. 3). Opistosoma olivastro, un poco più chiaro del prosoma; flagello, v. fig. 5, 8, 9; apparato genitale, v. fig. 4.

Dimensioni (in mm, leggermente approssimate): prosoma lungo 2,13 (dalla punta anteriore al margine posteriore del metapeltidio), largo 0,87 (propeltidio); opistosoma lungo 3,72 (fino alla punta del flagello). Lunghezza totale: 5,85.

\begin{tabular}{|c|c|c|c|c|c|c|}
\hline Zampe & Femore & Patella & Tibia & Basitarso & Tarso & Totale \\
\hline I & 2,77 & - & 4,16 & 3,02 & 1,64 & 11,59 \\
II & 1,46 & 0,85 & 1,06 & 0,87 & 0,60 & 4,84 \\
III & 1,16 & 0,57 & 0,72 & 0,52 & 0,68 & 3,65 \\
IV & 2,35 & 0,96 & 1,81 & 1,46 & 0,93 & 7,51 \\
\hline
\end{tabular}

Derivatio nominis: dedico con piacere questa specie al suo raccoglitore, Monsieur R. Pilet (Genève).

Discussione: LAWrence (1969), riprendendo varie osservazioni di HANSEN e SOERENSIN (1S05) ha proposto d risolvere l'annosa questione dei rapporti tra i generi Schizomus Cook, 1899 e Trithyreus Kraepelin, 1899 abolendo senz'altro Trithyreus. Non vi è alcun dubbio che in molti casi la divisione in due metà del metapeltidio può non essere evidente ed è anche vero che il significato di questo carattere è del tutto oscuro, ma è anche vero d'altronde che è estremamente improbabile l'esistenża di strette affinità tra tutte le specie di vari continenti riunite da LAWRENCE ed altri AA. sotto Schizomus.

La sistematica a livello generico degli Schizomidi è in pratica ancora tutta da fare; solo in tempi assai recenti alcuni AA. (Lawrence, Rowland) hanno proposto nuovi generi (oltre a Schizomus e Trithyreus) basandosi su caratteri di vario tipo (in generale forma del corpo, allungamento ecc.); il sistema risultante è però tutt'altro che omogeneo ed è assolutamente impossibile finora « costruire » dei gruppi di generi. Data questa situazione, assai insoddisfacente, abolire un nome è a mio parere prematuro. Non posso precisare se la nuova specie delle Batu Caves sia realmente prossima a Trithyreus grassii (Thorell, 1889), generotipo di Tripeltis Thorell, 1889 (cfr. THORELL, 1889: 554) e quindi generotipo di Trithyreus (nomen novum, cfr. KrAEPELIN, 1899: 234) visto che questa specie è stata descritta sulla femmina (ovviamente senza considerarne i genitali), ritengo però che la 


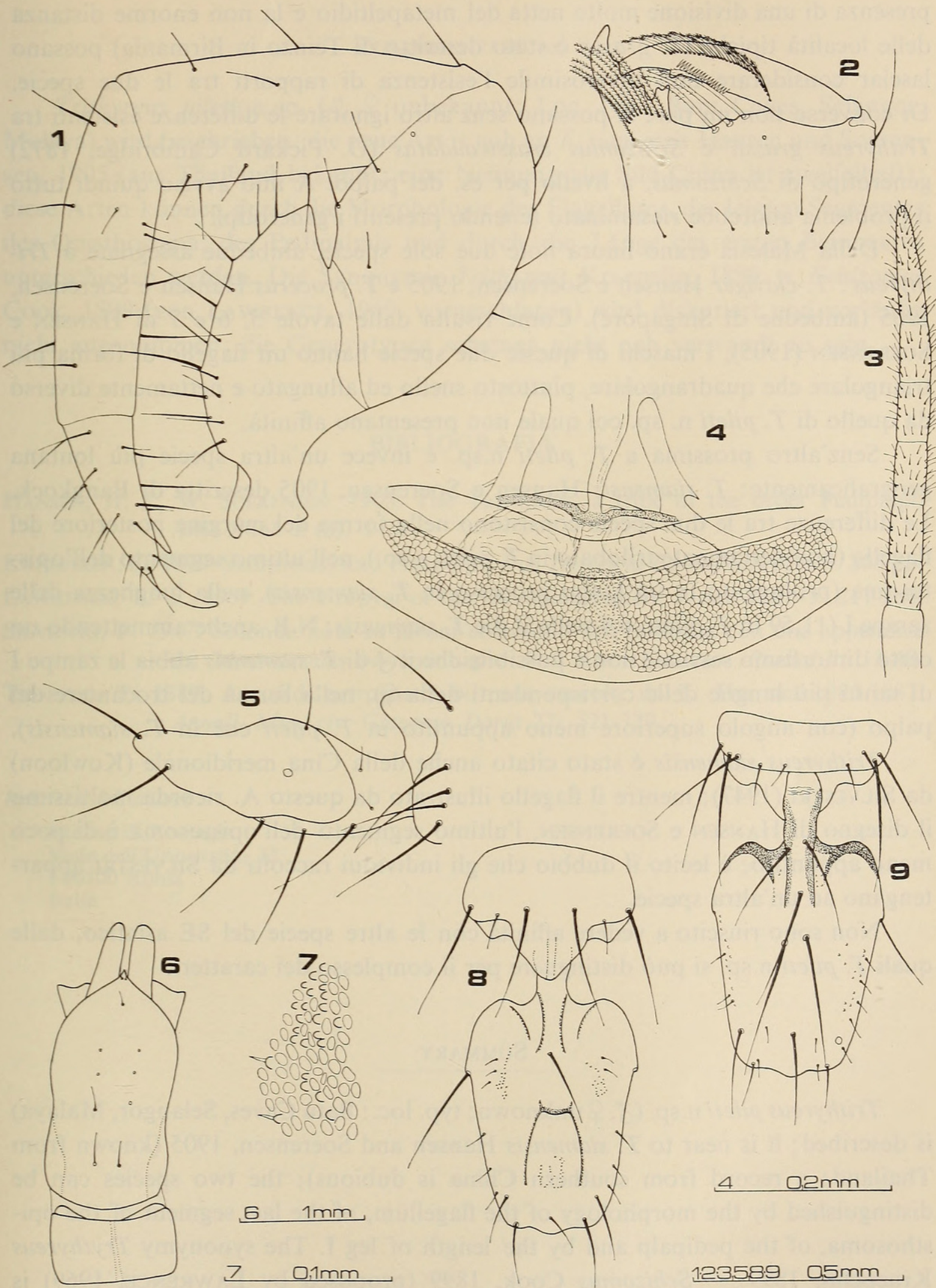

Trithyreus pileti $\mathrm{n}$. sp. - Fig. 1: palpo; fig. 2: estremità del chelicero; fig. 3: estremità del tarso I; fig. 4: apparato genitale; fig. 5, 8, 9: flagello (di fianco, da sopra e da sotto); fig. 6: prosoma; fig. 7: zona sensoriale (?) del flagello a forte ingrandimento. 
presenza di una divisione molto netta del metapeltidio e la non enorme distanza delle località tipiche ( $T$. grassii è stato descritto di Teinzo in Birmania) possano lasciar considerare non inverosimile l'esistenza di rapporti tra le due specie. Di converso non mi pare si possano senz'altro ignorare le differenze esistenti tra Trithyreus grassii e Schizomus crassicaudatus (O. Pickard Cambridge, 1872) generotipo di Schizomus, a livello per es. del palpo. A mio avviso quindi tutto il problema andrebbe riesaminato tenendo presenti i generotipi.

Della Malesia erano finora note due sole specie, ambedue assegnate a Trithyreus : T. claviger Hansen e Soerensen, 1905 e T. procerus Hansen e Soerensen, 1905 (ambedue di Singapore). Come risulta dalle tavole 5, 6 e 7 di HANSEN e SOERENSEN (1905), i maschi di queste due specie hanno un flagello di forma più triangolare che quadrangolare, piuttosto snello ed allungato e nettamente diverso da quello di $T$. pileti $\mathrm{n}$. sp. col quale non presentano affinità.

Senz'altro prossima a $T$. pileti n.sp. è invece un'altra specie più lontana geograficamente: T. siamensis Hansen e Soerensen, 1905 descritta di Bangkock. Le differenze tra le due specie consistono nella forma del margine posteriore del flagello (non nettamente trilobato in T. pileti n.sp.), nell'ultimo segmento dell'opistosoma (prolungato in un lungo processo in $T$. siamensis), nella lunghezza delle zampe I (11,59 in T. pileti, 6,4 nella 9 die $T$. siamensis; N.B. anche ammettendo un certo dimorfismo sessuale non è possibile che il $\hat{\jmath}$ di $T$. siamensis abbia le zampe I di tanto più lunghe delle corrispondenti della ()), nella forma del trocantere del palpo (con angolo superiore meno appuntito in $T$. pileti che in $T$. siamensis).

Trithyreus siamensis è stato citato anche della Cina meridionale (Kowloon) da Silvestri (1947); mentre il flagello illustrato da questo A. ricorda moltissimo il disegno di Hansen e SoERensen, l'ultimo segmento dell'opistosoma è di poco meno appuntito; è lecito il dubbio che gli individui raccolti da SILVESTRI appartengano ad un'altra specie.

Non sono riuscito a vedere affinità con le altre specie del SE asiatico, dalle quali $T$. pileti n.sp. si può distinguere per il complesso dei caratteri.

\section{SUMMARY}

Trithyreus pileti n.sp. (ô, o unknown; typ. loc.: Batu Caves, Selangor, Malaya) is described; it is near to T. siamensis Hansen and Soerensen, 1905 (known from Thailand; a record from southern China is dubious); the two species can be distinguished by the morphology of the flagellum, of the last segment of the opisthosoma, of the pedipalp and by the length of leg I. The synonymy Trythyreus Kraepelin, 1899 = Schizomus Cook, 1899 (proposed by LAwrENCE, 1969) is discussed and, for the moment, rejected as the generotypes do not seem strictly related. 


\section{ZUSAMMENFASSUNG}

Trithyreus pileti n.sp. ( $\hat{o}$, $q$ unbekannt; Loc. typ.: Batu Caves, Selangor, Malaya) wird beschrieben; die neue Art is nah an T. siamensis Hansen und Soerensen, 1905 (aus Thailand bekannt; eine Nennung aus Süd-China ist zweifelhaft); diese Arten können durch die Morphologie des Flagellums, des letzten Segmentes des Opisthosoma, des Pedipalpus und durch die Länge des ersten Beinpaares unterschieden werden. Die Synonymie Trithyreus Kraepelin, $1899=$ Schizomus Cook, 1899 (von LAWRENCE, 1969, vorgeschlagen) wird diskutiert und vorläufig nicht angenommen: die Generotypen scheinen nicht nah verwandt zu sein.

\section{BIBLIOGRAFIA}

Hansen, H. J., W. Soerensen. 1905. The Tartarides, a tribe of the order Pedipalpi. Ark. Zool. 2 (8): 1-78.

Kraepelin, K. 1899. Scorpiones und Pedipalpi. Das Tierreich, Berlin, 8, XVIII + 265 pp.

LAWrence, R. F. 1969. The Uropygi of the Ethiopian region. J. nat. Hist. 3: 217-260.

SilvestRI, F. 1947. Seconda nota su alcuni termitofili dell'Indocina con una appendice sul Macrotermes barneyi Light. Boll. Lab. Ent. agr. Portici 7: 13-40.

Thorell, T. 1889. Aracnidi artrogastri birmani raccolti da L. Fea nel 1885-1887. Annali. Mus. civ. Giacomo Doria 27: 521-729.

\section{Indirizzo dell'autore :}

Istituto di Zoologia

Viale dell'Università, 32

I-00100 Roma

Italie 


\section{$2 \mathrm{BHL}$ Biodiversity Heritage Library}

Brignoli, Paolo Marcello. 1974. "Un nuovo Schizomida delle Batu Caves in Malesia (Arachnida, Schizomida)." Revue suisse de zoologie 81, 731-735. https://doi.org/10.5962/bhl.part.76039.

View This Item Online: https://www.biodiversitylibrary.org/item/126812

DOI: https://doi.org/10.5962/bhl.part.76039

Permalink: https://www.biodiversitylibrary.org/partpdf/76039

\section{Holding Institution}

Smithsonian Libraries

\section{Sponsored by}

Biodiversity Heritage Library

\section{Copyright \& Reuse}

Copyright Status: In Copyright. Digitized with the permission of the rights holder.

Rights Holder: Muséum d'histoire naturelle - Ville de Genève License: http://creativecommons.org/licenses/by-nc-sa/3.0/

Rights: https://www.biodiversitylibrary.org/permissions/

This document was created from content at the Biodiversity Heritage Library, the world's largest open access digital library for biodiversity literature and archives. Visit BHL at https://www.biodiversitylibrary.org. 